\title{
Elementos de vulnerabilidade individual de adolescentes ao HIV/AIDS
}

\author{
Elements of adolescents' individual vulnerability to HIV/AIDS
}

Elementos de vulnerabilidad individual de adolescentes al VIH/SIDA

\section{Melina Mafra Toledo', Renata Ferreira Takahashi', Mónica Cecilia De-La-Torre-Ugarte-Guanilo' \\ 'Universidade de São Paulo. Escola de Enfermagem. São Paulo, SP}

Submissão: 08/09/2009

Aprovação: 15/07/2010

\section{RESUMO}

A adolescência é caracterizada pela experimentação e descoberta, pelo desenvolvimento da autonomia e pela sexualidade. O objetivo desse estudo foi identificar as evidências científicas da literatura sobre os elementos da dimensão individual da vulnerabilidade de adolescentes ao HIV/AIDS. Através da revisão sistemática integrativa, em bases e bancos de dados eletrônicos (Cinahl PubMed, Scopus, LILACS, Adolec, Dedalus, BDTD e Portal de Teses da Universidade de São Paulo) rastrearam-se trabalhos publicados entre 1996 e 2006. Compuseram a amostra final 41 estudos. O elemento da vulnerabilidade individual mais freQUente nos estudos foi o grau e Qualidade do conhecimento sobre HIV/AIDS. A revisão permitiu identificar evidências científicas da vulnerabilidade individual relevantes para o planejamento das ações de prevenção à infecção pelo HIV em adolescentes.

Descritores: Adolescente; HIV; Vulnerabilidade em saúde.

\begin{abstract}
Adolescence is characterized by experimentation and discovery, the development of autonomy and close search of sexuality. The objective of this study was to identify the scientific evidences of literature on the elements of the individual dimension of adolescents' vulnerability of to the HIV/AIDS. Through the integrative review, in electronic data bases (Cinahl PubMed, Scopus, LILACS, Adolec, Dedalus, Digital Library of Brazilian of Teses and Dissertações (BDTD) and Portal of Thesis of University of São Paulo), works published between 1996 and 2006 were tracked. 41 studies compose the final sample. The most frequent element of individual vulnerability in the studies was the degree and Quality of the knowledge on HI V/AIDS. The revision allowed identifying excellent scientific evidences of the individual vulnerability for the planning of the actions of prevention to the infection for the HIV in adolescents.
\end{abstract}

Key words: Adolescent; HIV; Health vulnerability.

\section{RESUMEN}

La adolescencia es caracterizada por la experimentación y descubrimiento, por el desarrollo de la autonomía y por la sexualidad. El objetivo de ese estudio fue identificar las evidencias científicas de la literatura sobre los elementos de la dimensión individual de la vulnerabilidad de adolescentes al VIH/AIDS. A través de la revisión sistemática integrativa, en bases y bancos de datos electrónicos (Cinahl PubMed, Scopus, LILACS, Adolec, Dedalus, BDTD e Portal de Teses da Universidade de São Paulo) fueran rastreados trabajos publicados entre 1996 y 2006. Compusieron la muestra final 41 estudios. El elemento de la vulnerabilidad individual más frecuente en los estudios fue el grado y calidad del conocimiento sobre HIV/AIDS. La revisión permitió identificar las evidencias científicas de la vulnerabilidad individual relevantes para la planificación de las acciones de prevención a la infección por el HIV en adolescentes.

Descriptores: Adolescente; VIH; Vulnerabilidad en salud.

\footnotetext{
AUTOR CORRESPONDENTE Melina Mafra Toledo. Escola de Enfermagem da USP. Avenida Enéas Carvalho de Aguiar, 4I9. CEP 05403-000.

São Paulo, SP. E-mail: melinamafra@usp.br
} 


\section{INTRODUÇÃO}

Atualmente, a infecção do vírus da imunodeûciência humana (HIV) representa um fenômeno global e dinâmico cuja ocorrência depende de uma rede complexa de determinantes políticos, econômicos, sociais e culturais. O percentual de infecções na adolescência torna-se mais significativo se considerarmos Que a AIDS se manifesta entre sete a dez anos após a infecção pelo HIV. Logo, supõe-se Que parte significativa das notificações da faixa etária de 25 a 29 anos corresponda a indivíduos Que se infectaram na adolescência ou no início da juventude ${ }^{(1)}$.

Dados epidemiológicos internacionais mais recentes apontaram a estimativa Que para o ano de 2007, em todo o mundo, $33 \mathrm{mi}-$ Ihões de pessoas vivam infectadas com o Vírus da Imunodeficiência Humana (HIV). Os jovens entre 15 e 24 anos representam atualmente $45 \%$ do total estimado das novas infecções por HIV em todo o mundo ${ }^{(2)}$.

No Brasil, a taxa de incidência (por 100.000 hab.) de casos de AIDS notificados no sexo masculino na faixa etária de 13 a 19 anos foi de 2,2 em 2008, para o mesmo ano e faixa etária a incidência foi de 2,8 entre o sexo feminino.O crescimento das notificações no sexo feminino foi maior se comparado ao sexo masculino, o Qual se manteve relativamente estável. A categoria de exposição heterossexual foi a principal causa de contaminação em ambos os sexos com indivíduos com 13 anos ou mais ${ }^{(3)}$.

Estudos demonstram Que muitos jovens estão iniciando sua vida sexual mais cedo (em média 15 anos para ambos os sexos) ${ }^{(4-7)}$ muitas vezes sem proteção contra gestações não planejadas e DST/ AIDS. Tal dado torna o percentual de infecções na adolescência mais significativo se considerarmos Que a AIDS se manifesta de sete a dez anos após a infecção pelo HIV. Logo, supõe-se Que grande parte das notificações, em pessoas com 25 a 29 anos corresponda a indivíduos Que se infectaram na adolescência ou no início da juventude, cujos parceiros sexuais também estiveram vulneráveis à infecção.

A educação sexual para adolescentes tem sido influenciada pelas políticas de governo e pela opinião pública. Ainda Que se apresentem pautadas em diferentes ideologias e estratégias, o sucesso dos programas é avaliado por promover mudanças comportáveis Que levem a práticas sexuais mais seguras ${ }^{(7)}$.

A crítica a essas abordagens educativas centradas na informação para a mudança de comportamentos mostra a necessidade de refletir não só sobre o conteúdo da informação, mas, principalmente, sobre como e por Que a informação é comunicada ${ }^{(8)}$.

Intervenções bem sucedidas Que produzam de fato recursos para a proteção dos adolescentes, devem ser pensadas e avaliadas de forma a considerar Que os problemas de saúde possuem componentes amplos e inter-relacionados, Que não podem ser avaliados e tratados de forma isolada. Pensar em estratégias de intervenções Que não somente definam comportamentos corretos para os demais, mas Que criem oportunidades de reflexão e interação dialógica entre sujeitos sociais, torna-se um convite desafiador.

O conceito de vulnerabilidade, emergiu no Brasil, em meados da década de 90 como possibilidade de interpretação da epidemia da AIDS e com a perspectiva de reconceituar a tendência de culpabilizar os indivíduos infectados, destacando-se os estudos de Ayres e colaboradores ${ }^{(9)}$. Segundo os autores o conceito pode ser sintetizado como "o movimento de considerar a chance de exposição das pessoas ao adoecimento como a resultante de um conjunto de aspectos não apenas individuais, mas também coletivos e contextuais"(10).

Segundo Takahashi ${ }^{(11)}$ um importante alcance do conceito é conferir maior integralidade às ações de saúde, pois defende propostas de intervenções Que consideram a interdependência dessas três dimensões citadas.

Portanto, a opção pela vulnerabilidade não é somente uma tentativa de aderir a um conceito ou refutar os demais, e sim, um esforço para entender o fenômeno AIDS em sua totalidade dinâmica e complexa.

O levantamento bibliográfico preliminar ao projeto desta pesQuisa permitiu constatar Que a maioria dos estudos sobre vulnerabilidade ao HIV centrava-se unicamente na dimensão do indivíduo, em comportamentos de risco ou traziam pouca ou nenhuma evidência sobre outros determinantes e condicionantes da doença.

Acredita-se Que a caracterização da vulnerabilidade à infecção pelo HIV, possibilita identificar seus elementos, os Quais por sua vez, contribuem para a proposição de intervenções voltadas para os reais determinantes da ocorrência da infecção. Dessa maneira, ter-se-á subsídios concretos, isto é, aderentes à realidade dos adolescentes, para o desenvolvimento de ações protetoras contra as condições Que determinam a ocorrência da infecção.

Com base no exposto realizou-se a presente revisão integrativa, com objetivo identificar as evidências científicas da literatura sobre os elementos da dimensão individual da vulnerabilidade de adolescentes ao HIV/AIDS. O objetivo deste artigo é caracterizar os estudos selecionados e apresentar os resultados focalizando os elementos da dimensão individual da vulnerabilidade de adolescentes ao HIV/AIDS.

\section{METODOLOGIA}

A Revisão Integrativa (RI) é assim denominada por fornecer informações mais abrangentes sobre um fenômeno ou problema de saúde interconectando elementos isolados de estudos já existentes.

Essa modalidade de revisão é definida como um método Que agrupa os resultados obtidos de pesQuisas primárias sobre o mesmo assunto, com o objetivo de sintetizar e analisar esses dados para desenvolver uma explicação mais abrangente de um fenômeno específico ${ }^{(12)}$. Realizada com rigor metodológico, contribui para o desenvolvimento da teoria e tem aplicabilidade direta nas práticas de saúde e na elaboração de políticas ${ }^{(13)}$.

Frente à diversidade de etapas para a realização da RI disponíveis na literatura, neste estudo optou-se pela proposta de Cooper ${ }^{(12)}$ devido à clareza na definição das etapas a serem seguidas, a saber: formulação da Questão norteadora da peseuisa, coleta dos dados, avaliação dos dados coletados, análise e interpretação dos dados e apresentação dos resultados.

A Questão norteadora do presente estudo foi "Quais os elementos considerados na caracterização e análise da vulnerabilidade individual dos adolescentes ao HIV/AIDS"?

Face ao vasto volume de informação disponível para a coleta de dados, foram utilizadas bases gerais, usuais em revisões sistemáticas na saúde e áreas afins e bases específicas direcionadas a temática do HIV/AIDS: Cummulative Index to Nursing and Allied Health 
Literature (Cinahl) - base internacional da produção da área de enfermagem), National Library of Medicine (PubMed - base multidisciplinar norte-americana), Scopus (base multidisciplinar com 53\% de seu conteúdo originário de países da Europa, América Latina e região Pacífica Ásia), Literatura Latino-Americana e do Caribe em Ciências da Saúde (LILACS), Saúde na Adolescência (ADOLEC base especializada em adolescentes). Além dessas bases adotou-se como fonte de dados: Banco de dados bibliográficos da Universidade de São Paulo (Dedalus), Biblioteca Digital de Teses e Dissertações da Universidade de São Paulo USP e a Biblioteca Digital Brasileira de Teses e Dissertações (BDTD) da Coordenação de Aperfeiçoamento de Pessoal de Nível Superior (Capes), contatos, por meio eletrônico, com alguns autores e busca por documentos e publicações no site oficial do Programa Nacional de DST/AIDS.

Os critérios de inclusão foram: produções científicas (artigos, teses e dissertações) Que abordassem algum elemento, já descrito, da vulnerabilidade de adolescentes de ambos os sexos soronegativos ao HIV/AIDS, estudos publicados em língua portuguesa, inglesa ou espanhola entre os anos de 1996 e 2006. A delimitação do recorte temporal da pesquisa teve por início o ano de 1996, ano Que iniciaram no Brasil as produções científicas sob a ótica do conceito de vulnerabilidade à AIDS. Por se tratar de um estudo com limite temporal definido para sua realização para busca optou-se por restringir a busca até o fim do ano de 2006.

O critério de inclusão, relativo à faixa etária correspondente a adolescência, teve como base a definição da OMS que considera o grupo com idade entre 10 e 19 anos. Estudos Que tinham como amostra este e outros segmentos etários deveriam apresentar os resultados por faixa etária para serem incluídos na revisão. Da mesma forma procedeu-se com os estudos desenvolvidos com grupos de adolescentes soronegativos ou soropositivos, sendo Que estes últimos seriam incluídos se estivesse descrito algum elemento de vulnerabilidade presente anteriormente à infecção.

$\mathrm{Na}$ fase de coleta de dados foram testadas duas estratégias de busca (A e B). Na estratégia A os descritores "HIV", "HIV Infections", "Aceuired Immunodeficiency Syndrome" e "Adolescent "foram pesquisados separadamente em todas as bases. Vulnerabilidade como foi utilizada como palavra do título, resumo ou texto, uma vez Que não foi encontrado nenhum descritor correspondente. $\mathrm{Na}$ estratégia B com o objetivo de reduzir a Quantidade e aumentar a especificidade dos trabalhos, como recomendam Bernardo, Nobre e latene ${ }^{(14)}$, foram incluídas na busca palavras referentes aos elementos da dimensão individual da vulnerabilidade a saber: modos de vida, conhecimento, atitudes e práticas em saúde, comportamento de saúde ou reprodutivo, prevenção primária. A estratégia A recuperou maior número de estudos pertinentes em todas as bases de dados Quando comparada à estratégia $\mathrm{B}$, sendo por isso eleita como melhor estratégia para busca para esta revisão.

O software Endnote ${ }^{\circledR}$ foi utilizado para o gerenciamento das referências bibliográficas pela facilidade de aplicação e disponibilidade na maioria das bases e periódicos. Auxiliou na importação e transferência direta dos estudos selecionados das bases pesQuisadas para um arquivo pessoal da pesquisadora.

Dentre o universo de 66l estudos Que tiveram os resumos lidos, foram pré- selecionados 65 estudos Que foram lidos leitura na íntegra. Foram realizadas reuniões de consenso com outra pesQuisadora Quando havia dúvidas sobre a inclusão de estudos na revisão e os estudos cujos resumos eram dúbios foram lidos na íntegra.

Para orientar a extração dos dados dos estudos elaborou-se uma ficha contendo dados relativos ao título, autoria, ano da publicação, metodologia, objetivos e resultados Que abordassem os elementos da vulnerabilidade e a decisão por incluir ou excluir o estudo.

Para avaliar a Qualidade metodológica dos estudos foi utilizado um instrumento modificado a partir do Critical Appraisal Skills Programme (CASP) ${ }^{(15)}$. Optou-se por este instrumento por permitir aos pesquisadores iniciantes avaliar a Qualidade dos estudos Quantitativos e Qualitativos de maneira objetiva, sistemática e ser de fácil operação. Os estudos foram classificados segundo a pontuação obtida na aplicação deste instrumento, sendo: A (06 a 10 pontos) os estudos com boa Qualidade metodológica e viés reduzido e, B (no mínimo 05 pontos), estudos com Qualidade metodológica satisfatória, mas com potencial de viés aumentado.

$\mathrm{Na}$ etapa de interpretação dos dados os elementos da dimensão individual identificados foram comparados com os já descritos por Ayres et $\mathrm{al}^{\left({ }^{(1)}\right)}$ visando destacar as semelhanças, diferenças ou novos elementos Que retratassem a vulnerabilidade de adolescentes. Frente à intersecção entre as evidências científicas relacionadas a cada elemento realizou-se um novo agrupamento destas, segundo três categorias temáticas seguintes: conhecimento, comportamento e percepção da vulnerabilidade

\section{RESULTADOS E DISCUSSÃO}

Dentre os 661 estudos identificados nas fontes de pesquisa, foram eliminados 596 por não atenderem aos critérios de inclusão. Na base Cinahl recuperou-se maior número de estudos, entretanto na base PubMed obteve-se o maior número de estudos selecionados.

A leitura na íntegra dos estudos revelou Que a utilização do descritor "adolescente" recuperou estudos que continham no título o descritor, todavia a população estudada incluía além de adolescentes, jovens e crianças sem discriminar os resultados e análises por faixa etária, não permitindo a extração dos resultados e as discussões referentes ao grupo de adolescentes. Da mesma forma os estudos mencionavam e/ou usavam o termo vulnerabilidade, entretanto a análise pautava-se no conceito de risco. Assim, após leitura na íntegra e análise do total de trabalhos pré-selecionados 4 I estudos constituíram a amostra final dessa revisão.

Foram analisados 38 artigos publicados em periódicos nacionais e internacionais, especializados (ou não) em adolescência e AIDS e três dissertações de mestrado. Na avaliação todos os estudos (100\%) foram classificados em nível A.

A maioria dos estudos foi de origem brasileira (Quatorze), seguidos de estudos de origem norte-americana (doze), africana (sete), asiática (três), oriente médio (três), australiana (um) e jamaicana (um).

A prevalência de estudos brasileiros dentre os países da América Latina pode ser justificada pela concentração de mais de um terço do total das pessoas vivendo com HIV no Brasil ${ }^{(16)}$. Além disso, a consolidação de esforços no enfrentamento da epidemia, seja pela definição de políticas públicas, operacionalizada pelo Programa Nacional de DST/AIDS, Que tornou o Brasil referência 
no âmbito da prevenção e controle do HIV para muitos países. Acresce-se a união de segmentos da sociedade na forma de organizações não-governamentais (ONG) e os recursos financeiros disponibilizados para pesquisas.

Quanto ao período de publicação, Quatro estudos situam-se na década de 1990, entre 1996 e 2000, e 37 entre 2001 e 2006. O grupo de estudos de 1996 e 2000, com exceção de um artigo Que trabalhou com profissionais de saúde, teve a abordagem focalizada no comportamento e conhecimento dos adolescentes sobre HIV/AIDS. Entre 2001 e 2006 observa-se Que embora muitos estudos ainda tivessem foco neste aspecto, as análises se ampliaram a outros campos de investigação, por exemplo, aos fatores socioculturais Que influenciavam os comportamentos, às desigualdades socioeconômicas e grupos específicos em situação de maior vulnerabilidade.

No tocante ao desenho dos estudos, vinte e três eram Qualitativos, doze Quantitativos, e seis estudos utilizaram a metodologia Qualitativa e Quantitativa simultaneamente.

Em relação à formação profissional do primeiro autor, médicos apresentaram a maior porcentagem $(24,4 \%)$, seguido de enfermeiros e de cientistas sociais em mesma proporção $(17,1 \%)$, psicólogos $(14,6)$, antropólogo $(4,9)$, assistente social $(2,4 \%)$. Sendo Que $19,5 \%$ (8) dos estudos não mencionaram a formação do autor. Nas publicações em Que não foi possível identificar a formação profissional dos autores, foram referidas suas instituições de origem, e todas estavam relacionadas à área de saúde ou ciências sociais.

A diversidade de formação dos profissionais, correspondente a várias subáreas de conhecimento das áreas da saúde e ciências sociais, retrata a complexidade da AIDS, a Qual requer o desenvolvimento de pesquisas, Que se complementem pela multiplicidade de abordagens, favoreça o diálogo interdisciplinar e permita uma melhor compreensão deste fenômeno.
O Quadro I contém os elementos da dimensão individual da vulnerabilidade a infecção pelo HIV de adolescentes elementos da dimensão individual da vulnerabilidade da de adolescentes ao HIV, distribuídos em dois grupos: no primeiro grupo estão expostos os elementos apresentados pelo conceito de vulnerabilidade proposto por Ayres et $\mathrm{al}^{(10)}$ e no segundo grupo apresentam-se os elementos de vulnerabilidade extraídos das evidências científicas dos estudos.

Quase todos os elementos extraídos dos estudos podem ser classificados como constituintes dos elementos apresentados no conceito de vulnerabilidade. O elemento Que compõe a vulnerabilidade, mais frequente nos estudos, foi o grau e Qualidade do conhecimento sobre HIV/AIDS. Os estudos revelaram conhecimento insuficiente sobre a infecção, ambivalência sobre o conhecimento e a adoção de práticas protetoras e comportamentos sexuais vulneráveis.

Muitos adolescentes não tinham o conhecimento correto ou suficiente a respeito dos comportamentos sexuais específicos de risco, por exemplo, não saber Que podiam se infectar por sexo oral/anal, ou durante o período menstrual ou Que o vírus pode ser transmitido durante a gestação ou no parto. Além disso, apresentaram equívocos Quanto às estratégias da prevenção Que não envolviam o uso do preservativo como as crenças sobre procedimentos Que poderiam protegê-los da infecção pelo HIV: praticar coito interrompido tomar um antibiótico, realizar uma ducha ou tomar uma vitamina após a relação sexual ${ }^{(17)}$.

Por outro lado, embora os adolescentes tivessem conhecimento acerca da transmissão e formas de prevenção à infecção, notou-se um descompasso entre o discurso e a prática de medidas de prevenção, de fato. Acreditar Que o preservativo "só precisava ser usado nas "transas" esporádicas, "com Quem não se conhece bem", separar com Quem irão transar e ter relacionamento sério, preferindo as virgens ou as garotas de "família" para não correr o risco

\begin{tabular}{|c|c|c|}
\hline \multicolumn{3}{|c|}{ DIMENSÃO INDIVIDUAL DA VULNERABILIDADE } \\
\hline Extraídos do conceito & \multicolumn{2}{|l|}{ No elementos: 16} \\
\hline \multicolumn{3}{|c|}{$\begin{array}{l}\text { Valores, interesses, crenças, credos, desejos, conhecimentos, atitude, comportamentos, relações familiares, relaçôes de } \\
\text { amizade, relaçôes afetivo-sexuais, relaçôes profissionais, situação material, situação psico-emocional, situação fisica, redes e } \\
\text { suportes sociais. }\end{array}$} \\
\hline Extraídos das evidências científicas & № elementos: 13 & \\
\hline $\begin{array}{l}\text { Tematica conhecimento } \\
\text { - grau e Qualidade do conhecimento } \\
\text { sobre HIV/AIDS; } \\
\text { - associação da transmissão do HIV a } \\
\text { "grupos de risco"; } \\
\text { - conhecimentos incorretos e/ou } \\
\text { insuficientes sobre prevenção e } \\
\text { transmissão do HIV; } \\
\text { - conhecimento das formas de } \\
\text { prevenção não incorporadas ao } \\
\text { cotidiano; } \\
\text { - AIDS "como doença do outro" } \\
\text { - aparecimento tardio dos sintomas da } \\
\text { infecção; }\end{array}$ & $\begin{array}{l}\text { Temática comportamento e seus } \\
\quad \text { condicionantes } \\
\text { - relações de gênero (confiança na } \\
\text { monogamia/ submissão ao parceiro) } \\
\text { dificuldade em negociar o uso do } \\
\text { preservativo } \\
\text { - incômodo ou atitudes negativas em } \\
\text { relação ao uso do preservativo; } \\
\text { - uso de preservativo em } \\
\text { relacionamentos sexuais esporádicos; } \\
\text { - prática do uso de preservativo como } \\
\text { método contraceptivo e não como } \\
\text { meio de prevenção ao HIV } \\
\text { - uso de drogas comprometendo a } \\
\text { adoção de práticas protetoras }\end{array}$ & $\begin{array}{l}\text { Temática percepção da vulnerabilidade } \\
\text { - ausência de percepção de sua } \\
\text { vulnerabilidade (não reconhece Que } \\
\text { possui práticas e comportamentos em } \\
\text { Que há exposição ao HIV); } \\
\text { - ausência de perspectivas de um futuro } \\
\text { promissor }\end{array}$ \\
\hline
\end{tabular}

Quadro 1. Dimensão individual de vulnerabilidade do adolescente ao HIV/AIDS. 
de "pegar alguma doença", são achados Que explicitam conhecimentos, crenças, valores culturais Que os tornam vulneráveis ${ }^{(18-19)}$.

A dificuldade e os incômodos relativos à negociação e ao uso do preservativo remetem a aspectos ideológicos e culturais Que influenciam substancialmente a maneira de pensar e se portar frente à vulnerabilidade ao HIV.

Confiar no parceiro pode significar Que só devem manter práticas sexuais com este. O comportamento feminino ainda se encontra vinculado à subalternidade na relação da mulher com o homem; Quando o relacionamento envolve o afeto, é comum a sensação ilusória de invulnerabilidade ${ }^{(20)}$, como se o amor garantisse "proteção" contra a infecção às DST/HIV. Esse pensamento permeia todos os grupos etários, mas pode ser particularmente problemático para os adolescentes, visto Que mantém relacionamentos geralmente instáveis e de curta duração(2I). Assim, a confiança não seria o sentimento apropriado para resguardá-los da infecção.

Podem se postular algumas hipóteses sobre as razões Que levam os adolescentes a não incorporarem o conhecimento sobre HIV/AIDS às práticas de prevenção. Uma delas, também presente como resultado deste estudo, é Que a não percepção dos adolescentes sobre sua vulnerabilidade ao HIV/AIDS, abordada em muitos estudos $^{(22-23)}$ foi considerada importante na não adoção de práticas preventivas ao HIV entre os adolescentes. Tais autores constataram Que os adolescentes possuem representações sociais da AIDS como sendo "doença do outro", "doença de adultos", de "gente velha" ou de "grupos de risco" identificados como homossexuais, prostitutas e usuários de drogas.

O conhecimento de Que os sintomas da AIDS aparecem tardiamente pode ser apontado como outro elemento da vulnerabilidade dos adolescentes ao HIV, visto Que a adoção do comportamento preventivo seria mais provável se as manifestações do agravo fossem imediatas. Uma vez Que a AIDS não é percebida como uma consequência imediata da relação sexual desprotegida, os adolescentes passam a considerá-la uma ameaça distante de seu cotidiano deixando a prevenção em segundo plano.

O consumo de álcool e drogas ilícitas por adolescentes, especialmente aqueles em situação de rua, como estratégia de sobrevivência para fugir da fome, da exclusão social foi identificado como condição Que os colocam mais vulneráveis a situações de risco, pois o estado alterado de consciência compromete a capacidade de decisão para o uso do preservativo "O pessoal da rua não usa camisinha não, toma droga, fica doidão, não dá moral e transa sem Querer saber de nada, não"(23).

Por outro lado, adolescentes da Mongólia, país em Que a discussão sobre saúde sexual ainda constitui um tabu, adolescentes sinalizaram que "é mais fácil usar preservativo Quando as luzes estão apagadas e Quando se está bêbado", pois sentem Que seus medos ou rejeições são desinibidos, tornando-os "valentes" para negociar e interagir sexualmente com as garotas ${ }^{(24)}$.

A mudança de comportamento é fruto de um processo complexo, envolve um conjunto de determinantes (classe social, idade, relações de gênero, valores, entre outros) e exige uma continuidade de ações e projetos do próprio serviço de saúde, da escola e da comunidade.
Logo a Qualidade do conhecimento e as desigualdades nas condições de acesso são elementos pontualizadores da vulnerabilidade.

A expectativa frustrada de ter um futuro promissor foi sugerida como um fator desestimulante à adoção de comportamentos protetores à infecção pelo HIV. Diante da situação econômica e social desfavorável, as oportunidades de obter melhor nível de educação, emprego e moradia estavam limitadas; em decorrência disso os adolescentes expressaram peQuena preocupação e empenho em prevenir-se contra o HIV, visto Que o risco e a Qualidade de vida são conceitos relacionados ao futuro ${ }^{(56)}$. Visão compartilhada por Peres $^{(26)}$ ao defender Que se os adolescentes não têm acesso ao mundo do trabalho regular, à saúde, à escola, suas possibilidades de realização, acabam ficando restritas, porque o clima psicológico de seu cotidiano fica comprometido pelas tensões e conflitos daí derivados Assim, a ausência de um projeto de vida constitui um elemento da vulnerabilidade. Os jovens de classes sociais mais favorecidas postergam o início de sua vida sexual e usam mais proteção porque têm acesso à informação e aos insumos, e também porQue sentem Que suas vidas têm valor, têm projetos para o futuro e existem outros suportes para ancoragem da auto-estima Que não apenas a realização sexual/amorosa ${ }^{(27)}$.

\section{CONSIDERAÇÕES FINAIS}

O estudo teve por objetivo identificar as evidências disponíveis na literatura científica sobre os elementos da vulnerabilidade individual de adolescentes ao HIV/AIDS, extraídos de investigações produzidas na última década.

A metodologia utilizada possibilitou a identificação de evidências dos elementos já descritos no conceito de vulnerabilidade Que norteou a pesquisa. Possibilitou também a inclusão de um elemento não definido previamente, o Qual foi ausência de perspectivas de um futuro promissor.

É importante lembrar Que o método empregado neste estudo é limitado em alguns aspectos, pois o uso rigoroso de descritores padronizados para a indexação ainda não é uma realidade mundial, e os erros de indexação (adolescentis versus adolescentes), dificultam o processo de localização das publicações. Essa é uma grande dificuldade para os pesquisadores Que desenvolvem revisões sistemáticas (viés de localização).

A adolescência constitui uma fase de maior vulnerabilidade à infecção pelo HIV não só pelas modificações biopsicossociais Que ocorrem, mas também pela necessidade Que o adolescente possui de explorar o novo e experimentar riscos.

Os dados apresentados ao longo do texto indicam Que a abordagem do sexo seguro entre adolescentes continua sendo necessária.

Dada a complexidade da prevenção do HIV, não se pode ter um olhar individual e particular sobre o problema, há Que se considerar a interface entre as dimensões da vulnerabilidade. Permanece o desafio de se buscar alternativas criativas Que contemplem os elementos de vulnerabilidade individual dos adolescentes, e Que façam sentido nos diferentes contextos sócio-culturais em Que os adolescentes estão inseridos e vivenciam sua sexualidade. 


\section{REFERÊNCIAS}

1. Ministério da Saúde (BR). Secretaria de Vigilância em Saúde. Programa Nacional de DST e AIDS. Manual de rotinas para assistência de adolescentes vivendo com HIV/AIDS. Brasília: Ministério da Saúde; 2006.

2. Organização Mundial de Saúde. Situación de la Epidemia Mundial de VIH. Informe Sobre La Epidemia Mundial de SIDA 2008. [citado em 2009 Mar 20]. Disponível em: http:// data.unAIDS.org/pub/GlobalReport/2008jc $1510 \_2008$ global_report_pp29_62_es.pdf

3. Ministério da Saúde (BR). Programa Nacional de DST/AIDS. Casos de AIDS identificados no Brasil, segundo frequência de faixa etária por ano notificação. Bol Epidemiol 2009; 4-5.

4. Aquino EML, Heilborn ML, Knauth D, Bozon M, Almeida MC, Araújo I, et al. Adolescência e reprodução no Brasil: a heterogeneidade dos perfis sociais. Cad Saúde Pública 2003; 19 (sup 2): 377-88.

5. Borges ALV, Schor N. Início da vida sexual na adolescência e relações de gênero: um estudo transversal em São Paulo, Brasil, 2002 Cad Saúde Pública 2005; 2 I (2): 499-507.

6. Manlove I, Ikramullah E, Terry-Humen E. Condom use and consistency among male adolescents in the United States. I Adolesc Health 2008; 43: 325-33.

7. Kurby DB, Laris BA, Rolleri LA. Sex and HIV education programs: their impact on sexual behaviors of young people throughout the world. I Adolesc Health 2007; 40: 206-17.

8. Meyer DE, Mello DF, Valadão MM, Ayres JRCM “Você aprende. A gente ensina?": Interrogando relações entre educação e saúde desde a perspectiva da vulnerabilidade. Cad Saúde Pública 2006; 22(6): 1335-42.

9. Ayres IRCM. Vulnerabilidade e prevenção em tempos de AIDS. In: Barbosa RM, Parker RG, organizadores. Sexualidade pelo avesso: direitos, identidade e poder. $34^{\mathrm{a}}$ ed. Rio de Janeiro: Relumé-Dumará/ABIA/IMS-UERJ; 1999. p. 59-72

10. Ayres IRCM. Risco, vulnerabilidade e práticas de prevenção e promoção da saúde. In: Campos GWS, Minayo MCS, Akerman M, Drumond Júnior M, Carvalho YM, organizadores. Tratado de Saúde Coletiva. Rio de Janeiro: Fiocruz/Hucitec; 2006, 353-95.

11. Takahashi RF. Marcadores de vulnerabilidade à infecção, adoecimento e morte por HIV e AIDS. [tese]. São Paulo: Escola de Enfermagem, Universidade de São Paulo; 2006.

12. Cooper HM. Integrating research: a guide for literature reviews. London: SAGE; 1990.

13. Whittemore R, Knalf $K$. The integrative review: update meth- odology. I Adv Nurs 2005; 52: 546-53.

14. Bernardo WM, Nobre MRC, Jatene FB. A prática clínica baseada em evidências. Parte II Buscando as evidências em fontes de informação. Rev Assoc Med Bras 2004; 50(1): 104-8.

15. University of Kent Templeman Library. Critical appraisal skills Ppogramme (CASP). Disponível em: http://library.kent.ac.uk/ library/info/subjects/healthinfo/critapprais. html

16. Joint United Nations Programm of HIV/AIDS, UNAIDS.Country profile for Brazil. [cited on 2008 Feb 16]. Available from: http:/ /www.unAIDS.org/en/CountryResponses/Countries/brazil.asp

17. Morrison-Beedy Di, Carey MP, Aronowitz T. Psychosocial correlates of HIV risk behavior in adolescent girls. I Obstet Gynecol Neonatal Nurs 2002; 32: 94-101.

18. Peres CA, Paiva V, Silveira F, Peres RA, Hearst N. Prevenção da AIDS com adolescentes encarcerados em São Paulo. Rev Saúde Pública 2002; 36(sup 4): 76-81.

19. Levinson RA, Sadigursky C, Erchak GM.The impact of cultural context on brazilian adolescents' sexual practices. Adolescence 2004; 39(154): 203-27.

20. De La Torre-Ugarte Guanilo MC. Vulnerabilidade feminina ao HIV: metassíntese [dissertação]. São Paulo: Escola de Enfermagem, Universidade de São Paulo; 2008.

21 . Hillier L, Warr D, Harrison L. "When you carry condoms all the boys thinks you want it": negociating competing discourses about safe sex. J Adolesc 1998; 2 I (2): 15-29.

22. Lichtenstein B. HIV Risk and healthcare attitudes among detained adolescents in rural Alabama. AIDS Patient Care and STD 2000; 14(3): 1 13-26.

23. Azevedo RLW. Frequência do uso do preservativo e percepção de vulnerabilidade para o hiv entre adolescentes. [citado em 2007 Mar 14]. Disponível em http://www.AIDScongress.net/ 7congresso.php

24. Dudley C. Does familiarity breed complacency? HIV knowledge, personal contact, and sexual risk behavior of psychiatrically referred Latino adolescent girls hispanic. I Behav Sci 2002; 24(3):53-368.

25. White RT Reconceptualizing HIV infection among poor black adolescent females: an urban poverty paradigm. Health Promot Prac 2002; 3(3): 302-12.

26. Peres VLA. Concepções de família em população de periferia urbana. In: Souza SMG, organizador. Infância, adolescência e família. Goiânia: Cânone; 2001. p. 17-230.

27. Villela WV, Doreto DT. Sobre a experiência sexual dos jovens. Cad Saúde Pública 2006; 22(1 I): 2467-72. 